\title{
Corrigenda
}

\section{Volume 37 (1941), 199-228}

'The fractional dimension theory of continued fractions'

\author{
By I. J. GOOD
}

Department of Statistics, Virginia Polytechnic Institute and State University, U.S.A.

The following corrections to the above-mentioned article were obtained jointly with Werner Fritsch in correspondence in 1953 and 1954. These corrections seem worth publishing because of the current interest in Hausdorff-Besicovitch fractional dimensions.
For
Read
p. 201, line 1
$0 \cdot 583$
0.585
line 4
$0 \cdot 417$
0.415
p. 205, THEOREM 11, above
$\alpha_{n}$
$\alpha_{k}$
p. 206, one line below $(3 \cdot 3)$
(3.2)
p. 210
The outline of the proof of Lemma 5(i) seems to be incorrect. A correct proof is available from the author.
p. 215
In the second half of the page the application of Lemma 2 is suspect, so TheOREM 8 is unproven. But the proof of ThEOREM 5 is valid because (12.2) does not require the conditions $a_{r} \leqslant \Phi(r) \quad(r=1,2, \ldots$, $\left.n_{0}-1\right)$.
pp. $216-217$
There is a gap in the proof of THEOREM 6. It was filled by Fritsch and details are available from the author.
p. 223 , top
p. 223, equation $(19 \cdot 12)$
p. 228 , line 4
Details are available from the author.
The power of $1-2 x-y$ should read $1-2 x$.
The second equation obviously follows from the first one and I cannot recall exactly what I had in mind. I must have intended that $x_{0}, \sigma$ and the function $\psi$ should be found simultaneously, but I failed to outline an approach. 